\title{
The Effects of External Scattering on the Improvement of Random Lasers
}

\author{
Peymaneh Rafieipour and Abbas Ghasempour Ardakani* \\ ${ }^{a}$ Department of Physics, Shiraz University, Shiraz, Iran \\ *Corresponding Author Email: aghasempour@ shirazu.ac.ir
}

Regular paper: Received: Jun. 1, 2020, Revised: Jan. 2, 2021, Accepted: Jan. 14, 2021, Available Online: Jan. 16, 2021, DOI: 10.29252/ijop.14.2.143

\begin{abstract}
The random laser (RL) emission characteristics can be improved by many different routes including either the material processing or optimizing the concentration of the relevant constituents. These routes can be very hard and even not practical in many cases, leaving us with the search of new schemes for the externally improvement of the random laser performance. In this paper, we suggest a simple approach for the externally enhancement of the random laser emission properties that can be applied in any designed transparent random lasing structures with single mode or multimode emission. This approach is based on using an adhesive tape in order to introduce an external scattering medium to the lasing structure and also return back the amplified leaking photons. For our investigated sample with nonresonant feedback, it is demonstrated that the emission intensity can be increased by a factor of 4.2 and the random laser threshold can be decreased by a factor of 1.8 .
\end{abstract}

KEYWORDS: double-sided tapes, external feedback, multiple light scattering, random lasers.

\section{I.INTRODUCTION}

Light amplification by stimulated emission of radiation does not always need a Fabry-Perot cavity to establish. It was shown in the late 19th century that strong light multi-scattering in a random amplifying medium can provide the essential optical feedback for realizing a bright laser light emission [1], [2]. Contrary to conventional lasers that are based on two wellaligned mirrors, both laser light and laser-like emissions are likely to occur in a random structure embedded into a gain medium, without the need of cumbersome and expensive technologies. These new light sources are renowned as random lasers (RLs), because the strength of randomness has a major impact on the light emission characteristics. Based on the strength of randomness, a multi-mode or singled-peak emission can be obtained from the random structure. If the random amplifying medium falls within the diffusion regime, light encounters subsequent scattering events, exhibits a random-walk diffusive propagation and finally exists the gain medium. In this case, the optical feedback is non-resonant because the interference effects and phase information are ignored. This kind of RL with a non-resonant feedback is characterized by a sudden collapse of the spontaneous emission spectrum towards the maximum frequency of the gain line-shape and a remarkable increase of the emission intensity, when the pump fluence approaches a threshold [1], [3]-[5]. Recently, it was demonstrated that this singled-peak emission with a relatively broad linewidth on the order of 4-9 $\mathrm{nm}$ is attributed to the coupling of many lasing modes oscillate simultaneously [6], [7]. On the other hand, random lasing emission with resonant feedback occurs in a random amplifying medium falling within the localization regime [2], [8]-[10]. In this situation, the transport scattering length of light is on the order of the emission wavelength. Therefore, light traps inside the gain medium and confines to closed 
loop paths via constructive interference events. Since the phase information is maintained in constructive interference events, there appear several lasing modes with linewidths less than $1 \mathrm{~nm}$ in the emission spectrum. During the past few years, different kinds of RLs have been fabricated, including plasmonic RLs [11], quantum dot RLs [12], fiber RLs [13], Raman RLs [14] and electrically pumped RLs [15]. The controllable spatial coherency of RLs [16], simple and low cost fabrication and no directionality are some of their noteworthy properties. As demonstrated in many research works reported in the literature [17], RLs can find potential applications in lightening, bioimaging, sensing, and display devices.

During the past years, the improvement of the RL emission characteristics has been the focus of many experimental studies. Many research works have examined this issue in an attempt to reduce the lasing threshold, to enhance the emission intensity or to increase the emission stability. Since the RL emission properties depend on the strength of light multiscattering, one can easily achieve the low lasing thresholds and high emission intensities by optimizing the concentration or randomness of the scattering constituents. Also, increasing the stability of the emission is possible by using organic dyes doped polymers instead of the frequently used organic dye counterparts [18]-[23]. In addition, choosing proper materials can play an important role in improving the RL emission properties. However, these methods are not practical in many cases, where the scattering elements are fixed or changing the randomness and material parameters results in high industrial and financial costs. Therefore, exploring new routes to externally enhance the lasing emission or externally reduce the lasing threshold is suggested. Since, it can provide a new simple and low cost platform for lowering the lasing threshold, enhancing the emission intensity and also increasing the stability. So far, a few research works have studied the influence of external feedback on the improvement of the RL emission characteristics [24]-[26]. Although introducing external elements to RLs has received much interest, there have been no new works since 2011. In a first approach in 1997, a movable dichroic mirror was used as the external element in a scattering and gain system that was consisted of rhodamine 640 perchlorate dye and $\mathrm{TiO}_{2}$ nanoparticles in a poly (methyl methacrylate)-hydroxy ethyl methacrylate mixture (75\%:25\%) [24]. The main effect of the enhanced feedback from the external mirror was the 5-fold reduction in the lasing threshold. Furthermore, it was demonstrated that the peak emission intensity increases and the full width at half maximum (FWHM) decreases when the distance between the dichroic mirror and the sample decreases. In the case of RLs with resonant feedback, a similar work was reported by Cao et al. in the year 1999 [25]. It was shown that using a movable Aluminum (Al) mirror at one side of the $\mathrm{ZnO}$ film or underneath the sample can strongly influence the modes, intensity and the RL threshold. An increase in the lasing intensity as well as a $50 \%$ reduction in the lasing threshold was observed, when the $\mathrm{Al}$ mirror was brought closer to the sample edge. In addition, it was shown that changing the distance between the sample and the $\mathrm{Al}$ mirror from bellow results in the appearance and disappearance of the lasing modes. Also, another approach based on depositing an Aluminum mirror on the back side of the PMMA film containing $\mathrm{Rh} 6 \mathrm{G}$ and $\mathrm{Ag}$ nanoparticles (NPs) was reported for providing external feedback in RLs [26]. It was shown that injecting back the leaking photons was the only role of $\mathrm{Al}$ mirror as the external feedback. In addition, a 5-fold reduction in the RL threshold and more than 20 times enhancement of the emission intensity was demonstrated, experimentally.

In this paper, we suggest a new simple and inexpensive but practical method to improve the RL emission properties. It is based on using a sticky tape with a rough surface as an external scattering medium for enhancing the optical feedback. We stick a double-sided adhesive tape underneath a conventional RL to understand how RL emission affected by the 
presence of adhesive tape. Our conventional RL sample consists of a glass microscope slide coated with the solution of rhodamine $\mathrm{B}(\mathrm{RhB})$ dye doped SU-8 epoxy based polymer which was introduced in our previous work for the first time. We use such gain medium here because of its solidity, better efficiency and photostability features. Also, we choose the glass microscope slide as the scattering medium, because it is readily available, easily handled and renewable. Also, it has fixed and unchangeable scattering centers. We have demonstrated in our previous work that light multi-scattering from the surface roughness of the glass substrate can provide the necessary optical feedback for random lasing emission [27]. In this paper, we demonstrate that a sticky tape as a scattering medium can act as an external feedback element for improving the RL performance. When this adhesive tape is stuck on the backside of a conventional RL such as one reported in ref [27], the RL emission intensity increases while the lasing threshold decreases. Here, it is shown that using adhesive tape in RLs leads to the enhancement of RL emission intensity by a factor of 4.2 and the reduction of RL threshold by a factor of 1.8. Furthermore, the dependence of the random lasing spectrum on the excitation position is examined, experimentally. This novel work provides a way to enhance the emission of RLs and reduce their threshold after fabrication provided the RL sample is transparent enough such that its emission reaches their backside. In our previous work [28], we use a double sided adhesive tape as a scattering element in RLs and the gain medium is directly deposited on the tape. However, in this work the gain medium is coated on a glass substrate and the roughness existing in the surface of the substrate provides the multiple scattering in the RL system. Thus, here, the double sided tape is separated from the gain medium and only provides an external feedback to improve the random lasing features.

The remaining parts of the paper are organized as follows: a description of the experimental set up and sample preparation is presented in section 2. Section 3 is devoted to the experimental results and related discussions. Finally, the paper is finished with some conclusions in section 4 .

\section{Materials ANd MethodS}

\section{A. Ransom Laser Experiment}

To perform the experimental measurements and analyze the RL emission spectra, we used a set up that is illustrated in Fig. 1, schematically.

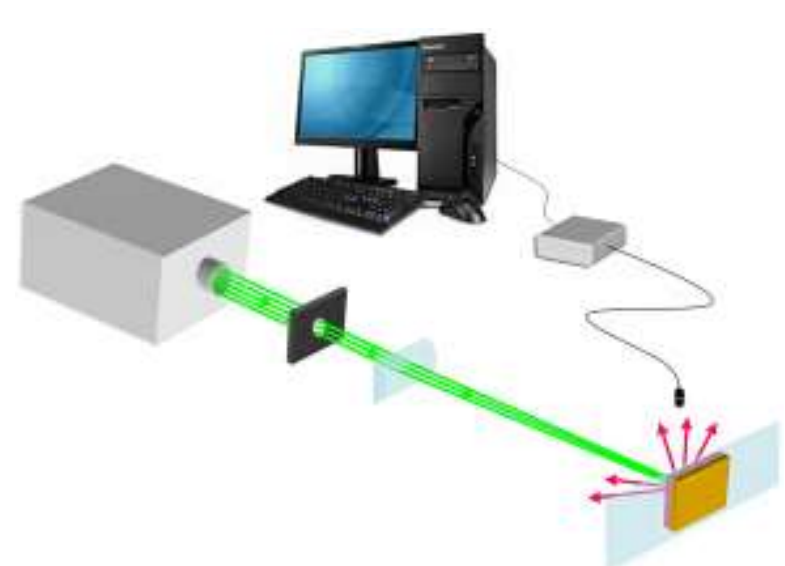

Fig. 1. Schematic illustration of the experimental set up. Sizes and distances are not scaled.

A neodymium doped yttrium aluminum garnet (Nd-YAG) laser with pulsed operation (10 ns and $10 \mathrm{~Hz}$ ) at $532 \mathrm{~nm}$ was used as the excitation source. The pump light was focused to a stripe with $8.95 \mathrm{~mm}$ length and $0.35 \mathrm{~mm}$ width on the sample, by using a cylindrical lens. In addition, an aperture diaphragm was placed in front of the pump laser to control and adjust the excitation area on the sample. The sample was a glass microscope slide $(76.45 \mathrm{~mm} \times 25.05 \mathrm{~mm} \times 1.05 \mathrm{~mm})$ that was stuck to a double sided tape from the back side. The sample was placed on a xyz-translation stage in order to be excited at different positions. An optical fiber was used to collect the emitted light from the sample. Then, the collected light was coupled to a spectrometer (ocean optics, the resolution of $0.1 \mathrm{~nm}$ ) and the corresponding emission spectrum was recorded and analyzed, by using a computer. 


\section{B. Sample Preparation}

The gain medium was prepared by dissolving $21 \mathrm{mg} \mathrm{RhB}$ dye (Aldrich) in $5 \mathrm{ml}$ solution of SU-8 epoxy based polymer, similar to the procedure described in [27]. Fig. 2 (a) depicts the preparation method of the investigated sample, schematically. This sample was composed of a glass microscope slide that was drop coated by the solution of RhB dye doped SU-8 epoxy based polymer. In this method, a few droplets of the gain solution were placed onto the surface of the glass substrate, by using a small dropper. The thickness of the gain layer was approximately 50 micron. The prepared sample, signed as S1 (sample 1), was left in the ambient temperature for 24 hours before performing the RL experiments.

(a)

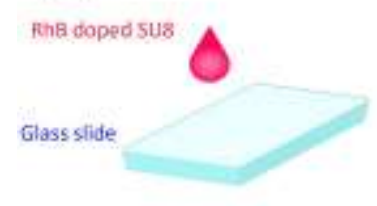

(c)

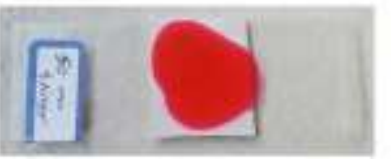

Fig. 2. Schematic illustration of the preparation method, by using drop coating technique: (a) RL sample (sample S1), (b) RL sample with a doublesided tape that is stuck to the back side of the sample (sample S2), (c) A camera photograph of the prepared sample $\mathrm{S} 2$.

As it is schematically illustrated in Fig. 2 (b), we placed an adhesive tape $(1.5 \mathrm{~mm}$ thick and $22 \mathrm{~mm}$ width) underneath the previously prepared sample and stuck it by a mechanical pressure. The morphology analysis and the scanning electron microscope (SEM) images of the tape are presented in our previous work [28]. The prepared new sample was then named as S2 (sample 2) and its RL emission characteristics was investigated and compared with that of S1. Figure 2 (c) displays a camera photograph of the prepared sample (S2).

\section{III.RESULTS AND DISCUSSION}

We start with the study of random lasing oscillation in a glass substrate coated with $\mathrm{RhB}$ dye doped SU-8 epoxy based polymer (sample $\mathrm{S} 1)$. The emission spectra of S1 are measured at different values of the pump fluence. The variation of the emission spectra as a function of the pump fluence is depicted in Fig. 3. The peak appearing at $532 \mathrm{~nm}$ is attributed to the wavelength of the excitation source. The lasing peak is located around $633 \mathrm{~nm}$.

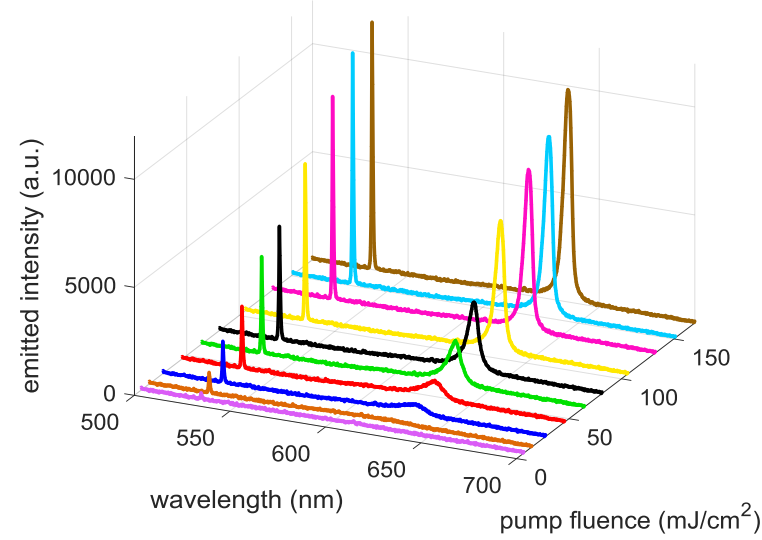

Fig. 3. Pump dependent variation of the emission spectrum corresponding to the glass substrate coated with $\mathrm{RhB}$ dye doped SU-8 epoxy based polymer (sample $\mathrm{S} 1$ ).

At very low pump fluences, we observe very broad emission spectra with the central wavelengths of approximately $626 \mathrm{~nm}$ that are ascribed to the spontaneous emission of the gain medium. For example, at the pump fluence of $14.4 \mathrm{~mJ} / \mathrm{cm}^{2}$, the linewidth and the central wavelength of the spontaneous emission spectrum are approximately $64 \mathrm{~nm}$ and $626.8 \mathrm{~nm}$, respectively. Although the spontaneous emission spectrum is dominant for very low pump fluences, it is narrowed and intensified by increasing the pump fluence, implying a threshold behavior for random lasing oscillation. One can see in Fig. 3 that the output intensity increases significantly at higher pump fluences. In addition, the linewidth of the emitted light is considerably reduced at very high pump fluences. At the pump fluence of $169.8 \mathrm{~mJ} / \mathrm{cm}^{2}$, the central wavelength and linewidth of the emission peak 
are approximately $633.7 \mathrm{~nm}$ and $5.1 \mathrm{~nm}$, respectively. The reason for these behaviors is the reduction of the gain length of light by the increase of the pump fluence [29]. Considering the RL theory with non-resonant feedback, a drastic increase of the emission intensity occurs when the gain length approaches the average path length of the emitted light inside the amplifying random medium. This phenomenon is accompanied by a remarkable collapse of the emission linewidth toward the central frequency of the gain line-shape. The role of the randomness in the diffusive amplifying medium is to increase the average path length of the emitted light inside the gain medium, resulting in the enhancement of the light emission intensity and the reduction of the lasing threshold.

These observations imply a threshold behavior corresponding to the investigated sample. One can estimate the RL threshold from Fig. 4, which displays the variation of the maximum emitted intensity in arbitrary units and the linewidth versus the pump fluence.

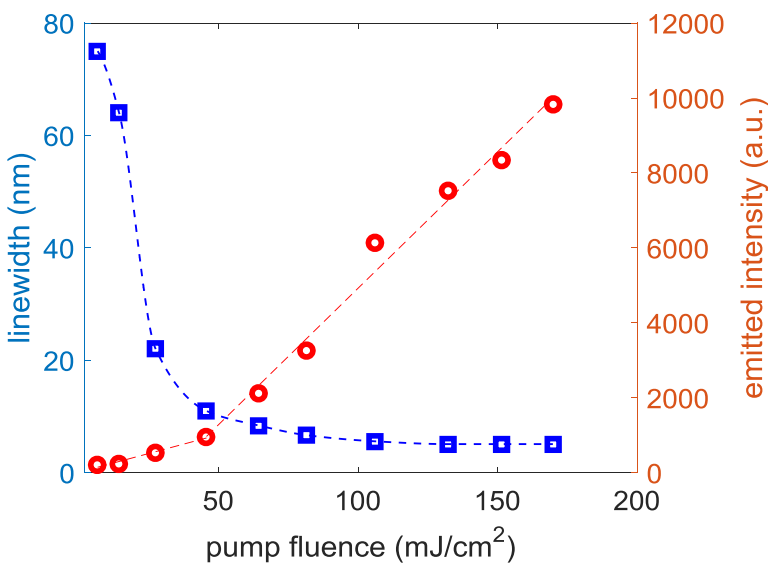

Fig. 4. Peak emitted intensity in arbitrary units, and linewidth of the emission peak versus the pump fluence, corresponding to the glass substrate coated with RhB dye doped SU-8 epoxy based polymer (sample S1).

The threshold pump fluence is the pump fluence at which a sudden increase in the emission intensity and a remarkable decrease in the emission linewidth occur. As it is shown in Fig. 4, the laser threshold is approximately $45.7 \mathrm{~mJ} / \mathrm{cm}^{2}$. In addition, the emission intensity increases with a faster slope, for pump fluences above the threshold. The linewidth also remains nearly constant far above the threshold pump fluence. As it was demonstrated in [27], light multi-scattering from the surface roughness of the glass substrate can be the physical mechanism providing the necessary optical feedback for the realization of random lasing action. These observations imply the occurrence of random laser action in the glass substrate coated with the solution of RhB dye doped SU-8 epoxy based polymer.

Next, we change the excitation position on the sample and measure the emission spectra at different pump fluences. Fig. 5 compares the emission spectra corresponding to two different excitation positions which are signed as P1 (position 1) and P2 (position 2) at the pump fluence of $169.8 \mathrm{~mJ} / \mathrm{cm}^{2}$.

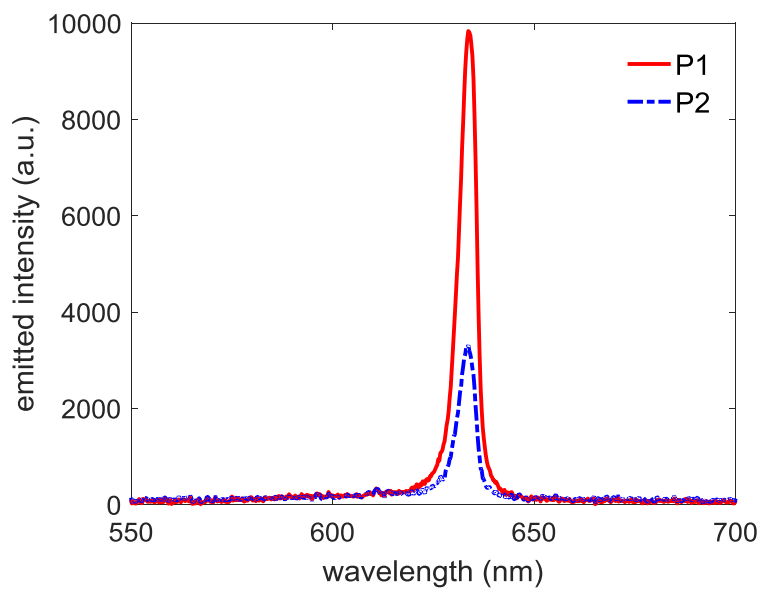

Fig. 5. Comparison between the emission spectra of S1 (sample 1) corresponding to the two different excitation positions P1 (position 1) and P2 (position 2) at the pump fluence $169.8 \mathrm{~mJ} / \mathrm{cm}^{2}$.

The output intensity at the excitation position P2 is considerably lower than that at the excitation position $\mathrm{P} 1$.The reason might lie in the higher amount of optical amplification that light gains at P1 compared with P2. It may be because of the relatively non-uniform surface of the gain medium on the glass substrate that results in different number of dye molecules existing at the excitation positions P1 and P2. Furthermore, the randomness of the glass 
surface can affect the strength of light multiscattering and so the optical feedback. It then results in different mean free paths of light at the excitation positions $\mathrm{P} 1$ and P2. Fig. 6 depicts the atomic force microscope (AFM) line height plot of the glass surface. The average height of the surface roughness is approximately $1.507 \mathrm{~nm}$.

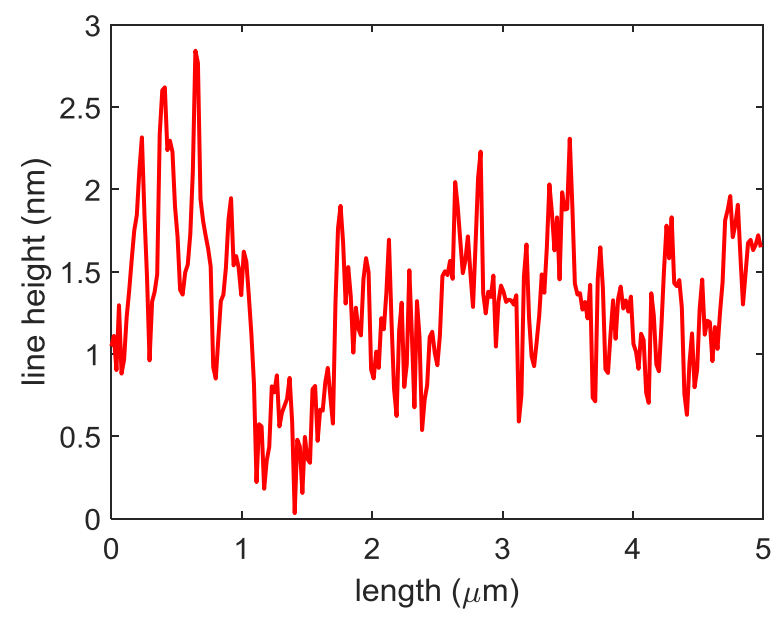

Fig. 6. Atomic force microscope (AFM) line height plot of the glass substrate (microscope slide).

It is inferred from Fig. 6 that the randomness strength corresponding to different excitation positions is different. As a result, the strength of light multi-scattering increases at regions with higher level of randomness. Hence, one can deduce that the emitted light at P1 undergoes more scattering events and gains higher amplification. It is then expected that the lasing condition at P1 fulfills at lower pump fluences and the corresponding RL threshold decreases.

The plots of the maximum emitted intensity and linewidth as a function of the pump fluence corresponding to the excitation positions $\mathrm{P} 1$ and $\mathrm{P} 2$ are depicted and compared with each other in Fig. 7. In this figure, circles and squares represent the experimental data of $\mathrm{P} 1$ and $\mathrm{P} 2$, respectively.

One can see in Fig.7 that the lasing oscillation triggers at a lower threshold pump fluence, when P1 is illuminated by the pump pulse. Its corresponding random laser threshold is

approximately $45.7 \mathrm{~mJ} / \mathrm{cm}^{2}$. Contrary to $\mathrm{P} 1$, the RL light shines at a higher laser threshold (approximately $68.0 \mathrm{~mJ} / \mathrm{cm}^{2}$ ) for the case in which $\mathrm{P} 2$ is illuminated by the pump pulse. Also, corresponding results indicate that the slope efficiency and the output intensity increase at the excitation position $\mathrm{P} 1$ with respect to $\mathrm{P} 2$. Consequently, the random lasing emission is dependent on the excitation position of the sample, consisting with the theory of RLs.
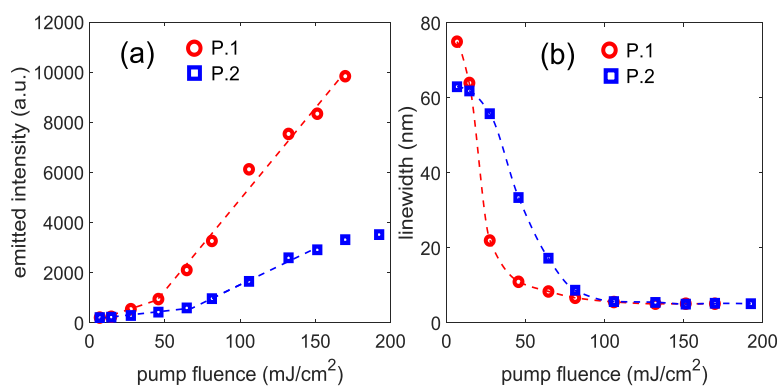

Fig. 7. Comparison between the pump dependent variations of: (a) maximum emitted intensity, and (b) linewidth, corresponding to S1 (sample 1) at different excitation positions $\mathrm{P} 1$ and $\mathrm{P} 2$.

In order to verify the role of using a doublesided adhesive tape as an external scattering medium in enhancing the RL performance, we measure the emission spectra corresponding to S2 at different pump fluences. The pump dependent variation of $\mathrm{S} 2$ for the cases in which the positions P1 and P2 are illuminated by the pump pulse are shown in Figs. 8 (a) and (b), respectively.

(a)

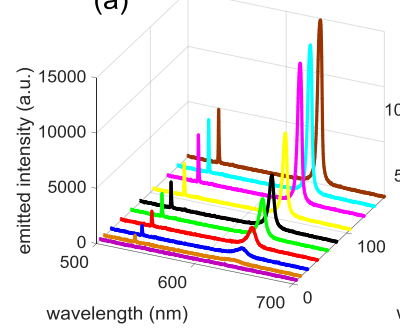

Fig 8. Pump dependent variation of the emission spectrum corresponding to S2 (sample 2) at: (a) the first excitation position $\mathrm{P} 1$, and (b) the second excitation position $\mathrm{P} 2$. 
The peak appearing at $532 \mathrm{~nm}$ exhibits the pump wavelength. At the low pump fluence $14.4 \mathrm{~mJ} / \mathrm{cm}^{2}$, the central wavelength and linewidth of the spontaneous emission spectrum are approximately 632.1 and $17 \mathrm{~nm}$, respectively for the case in which $\mathrm{P} 1$ is illuminated by the pump pulse. In addition, the spontaneous emission spectrum with the central wavelength of $626.8 \mathrm{~nm}$ and linewidth of $40.6 \mathrm{~nm}$ is observed for the excitation position $\mathrm{P} 2$ at the pump fluence $14.4 \mathrm{~mJ} / \mathrm{cm}^{2}$. It then intensified and narrowed by increasing the pump fluence. At the pump fluence of $151.3 \mathrm{~mJ} / \mathrm{cm}^{2}$, the central wavelength of the emitted peak corresponding to the excitation positions $\mathrm{P} 1$ and $\mathrm{P} 2$ are approximately 635 and $633.7 \mathrm{~nm}$, respectively. Also, the linewidths of the emitted peak corresponding to the excitation positions $\mathrm{P} 1$ and $\mathrm{P} 2$ are approximately $5.1 \mathrm{~nm}$, for the pump fluence of $151.3 \mathrm{~mJ} / \mathrm{cm}^{2}$. Although the variation of the emission spectrum as a function of the pump fluence is similar to $\mathrm{S} 1$ illustrated in Fig. 3, the intensity of the pump light reflected onto the fiber is considerably reduced. The appearance of the pump light in the emission spectra can be due to the light reflection by either the sample surface or sample holder. The role of the tape stuck to the back side of the sample in reducing the reflection of the pump light onto the fiber is to prevent the transmission of light through the sample in one hand and randomize the direction of the scattered light from the surface of the tape, on the other. Furthermore, the emission spectra of S2 exhibit intensive and narrow-band emission at both excitation positions $\mathrm{P} 1$ and $\mathrm{P} 2$.

We compare the emission spectra of S2 corresponding to the excitation positions $\mathrm{P} 1$ and $\mathrm{P} 2$ in Fig. 9, when the pump fluence is $151.3 \mathrm{~mJ} / \mathrm{cm}^{2}$. It is inferred that the output intensities are nearly comparable with each other and are enhanced for both the excitation positions $\mathrm{P} 1$ and $\mathrm{P} 2$. However, the emission intensity at P1 is slightly higher than P2 because of the reasons discussed in the previous paragraphs.

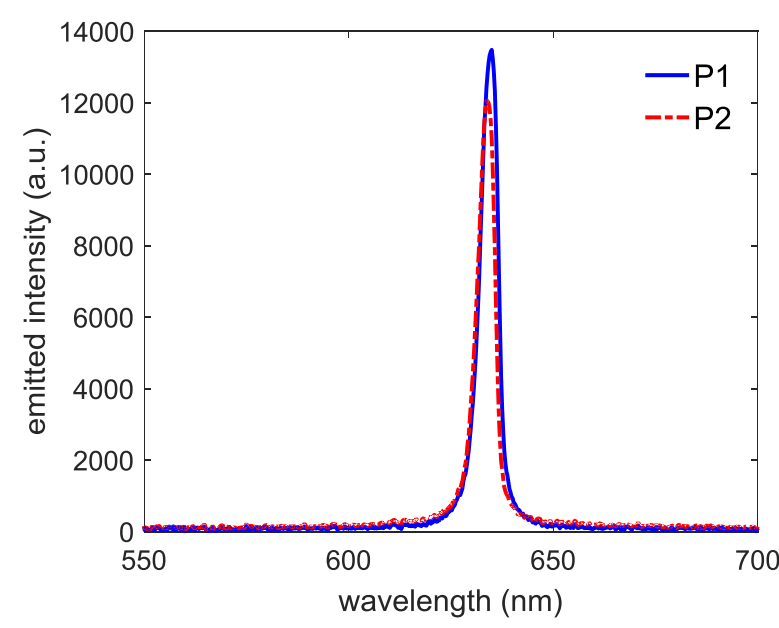

Fig. 9. Comparison between the emission spectra of S2 (sample 2) corresponding to two different excitation positions $\mathrm{P} 1$ (position 1) and P2 (position 2) at the pump fluence of $151.3 \mathrm{~mJ} / \mathrm{cm}^{2}$.

Moreover, the threshold behavior corresponding to $\mathrm{S} 2$ at the excitation positions $\mathrm{P} 1$ and $\mathrm{P} 2$ are compared with each other in Fig. 10. The experimental data corresponding to the excitation positions $\mathrm{P} 1$ and $\mathrm{P} 2$ are depicted by the squares and circles, respectively.
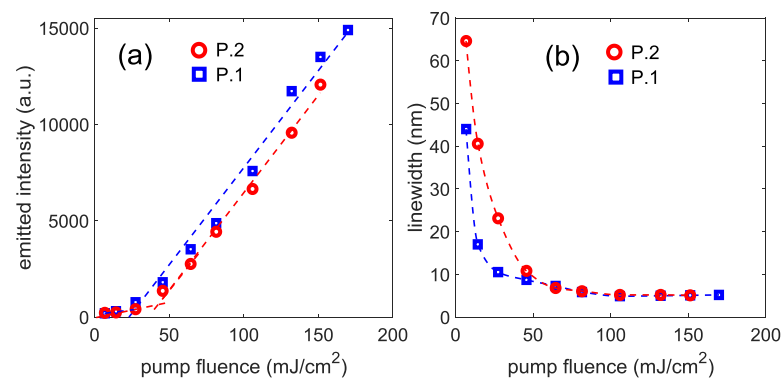

Fig. 10. Comparison of the pump dependent variations of: (a) maximum emitted intensity, and (b) linewidth corresponding to S2 (sample 2) at two different excitation positions P1 (position 1) and P2 (position 2).

The RL threshold values corresponding to the excitation positions $\mathrm{P} 1$ and $\mathrm{P} 2$ are approximately 27.5 and $38.0 \mathrm{~mJ} / \mathrm{cm}^{2}$, respectively. One can see that the RL thresholds are different, similar to that of S1. Nevertheless, it is clearly seen that the existence of the tape lowers the RL threshold. To better understand and visualize the effects of the double-sided tape as the external scattering medium in enhancing the RL 
performance, in the following, we will compare the emission spectra and the RL thresholds of S1 and S2 at each excitation positions $\mathrm{P} 1$ and $\mathrm{P} 2$, in one figure. It is noteworthy to mention here that the excitation positions $\mathrm{P} 1$ and $\mathrm{P} 2$ are the same when investigating the RL emission spectra from $\mathrm{S} 1$ and $\mathrm{S} 2$.

Figures 11 (a) and (b) compare the emission spectra of $\mathrm{S} 1$ and $\mathrm{S} 2$ corresponding to different excitation positions $\mathrm{P} 1$ and $\mathrm{P} 2$, respectively at the same pump fluence of $151.3 \mathrm{~mJ} / \mathrm{cm}^{2}$.
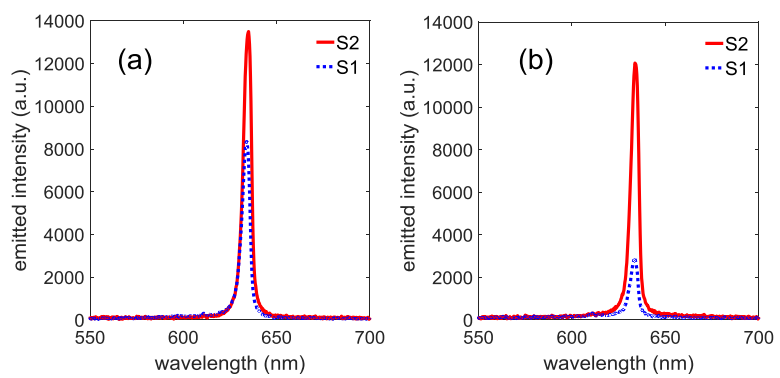

Fig. 11. Comparison between the emission spectra of the samples S1 and S2 at the excitation positions: (a) P1 and (b) P2. The pump fluence is 151.3 $\mathrm{mJ} / \mathrm{cm}^{2}$.

It is observed that the RL output intensity enhances, considerably by using a doublesided tape as the external scattering medium. It is worth mentioning that the enhancement factor of the emission intensity is different for different pump fluences, due to the energy fluctuations of the pump pulse. The output intensity enhances, on average, by a factor of approximately 1.50 and 4.22 at the excitation positions $\mathrm{P} 1$ and $\mathrm{P} 2$, respectively. Although the enhancement factor is larger for the position P2 compared with $\mathrm{P} 1$, the enhanced output intensities are nearly the same (see also Fig. 9). It is because the tape scatters back the leaking photons and increase the photon path lengths inside the gain medium. Hence, the system loss suppresses and the light gains more amplification. As a result, the output intensity increases. It is then suggested that the lasing threshold decreases for the sample with a double-sided tape (sample S2).
Figures 12 (a) and (b) display and compare the threshold behaviors of $\mathrm{S} 1$ and $\mathrm{S} 2$ corresponding to the excitation position P1, respectively. Also, Figs. 13 (a) and (b) show and compare the threshold behaviors of $\mathrm{S} 1$ and $\mathrm{S} 2$ corresponding to the excitation position $\mathrm{P} 2$, respectively. Circles and squares exhibit the experimental data of S1 and S2, respectively.
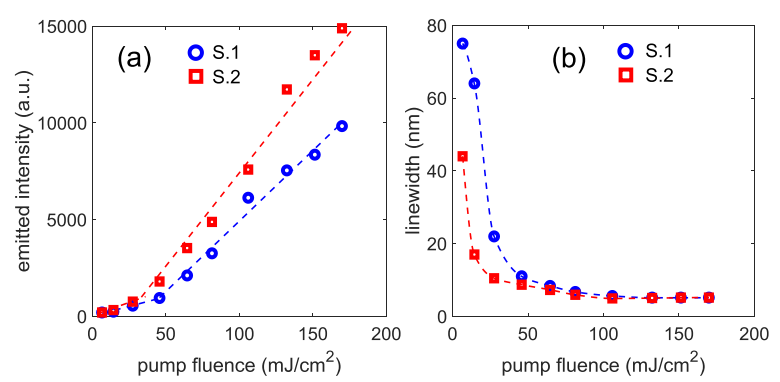

Fig. 12. Comparison between plots of: (a) maximum emitted intensity in arbitrary units, and (b) linewidth versus the pump fluence corresponding to the samples $\mathrm{S} 1$ and $\mathrm{S} 2$ at the excitation position $\mathrm{P} 1$.
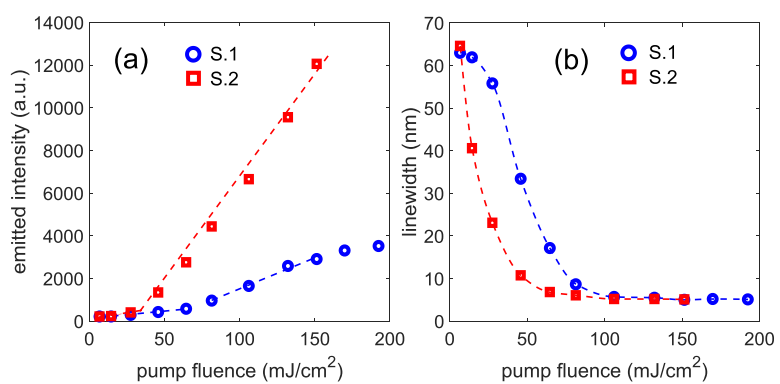

Fig. 13. Comparison between plots of: (a) maximum emitted intensity in arbitrary units, and (b) linewidth versus the pump fluence corresponding to the samples $\mathrm{S} 1$ and $\mathrm{S} 2$ at the excitation position $\mathrm{P} 2$.

The remarkable reduction in the RL threshold can be established for $\mathrm{S} 2$ in both the excitation positions $\mathrm{P} 1$ and $\mathrm{P} 2$. The $\mathrm{RL}$ threshold is reduced by factors of approximately 1.6 and 1.8 for the excitation positions $\mathrm{P} 1$ and $\mathrm{P} 2$, respectively. Obtained results are consistent with the previously reported experiments on the externally enhancement of the RL operation [24]-[26]. Although the enhancement factor in our proposed system is lower than that reported by others, using a double-sided tape as the external scattering medium may be advantageous from the point of simplicity, reliability and cost effective 
employment. Consequently, the double sided tape can be stuck on the backside of each RL cuvette cell or a transparent substrate of RLs to obtain stronger output emissions with a lower lasing threshold.

\section{CONCLUSION}

As a conclusion, we demonstrated a new scheme for promoting the RL operation by introducing an external feedback which was provided by a double-sided tape. The investigated RL sample was fabricated based on a glass substrate coated with $\mathrm{RhB}$ dye doped SU-8 epoxy based polymer. The RL action occurs in a glass substrate due to light multi-scattering from the roughness of the glass surface. The dependence of the lasing spectra on the excitation position was confirmed experimentally. Furthermore, it is demonstrated that sticking the back side of the sample to a double-sided tape enhances the output intensity and reduces the RL threshold, significantly. We hope that this work can provide a new simple approach for the enhancement of the conventional RL performance.

\section{ACKNOWLEDGMENT}

The authors are grateful to Dr. G. M. Parsanasab for his help with AFM measurements.

\section{REFERENCES}

[1] N.M. Lawandy, R.M. Balachandran, A.S.L. Gomes, and E. Sauvain, "Laser action in strongly scattering media," Nature, Vol. 368, pp. 436-438, 1994.

[2] H. Cao, Y.G. Zhao, S.T. Ho, E.W. Seelig, Q.H. Wang, and R.P.H. Chang, "Random laser action in semiconductor powder," Phys. Rev. Lett. Vol. 82, pp. 2278-2281, 1998.

[3] W.L. Sha, C.H. Liu, and R.R. Alfano, "Spectral and temporal measurements of laser action of Rhodamine 640 dye in strongly scattering media," Opt. Lett. Vol. 19, pp. 1922-1924, 1994.
[4] R.M. Balachandran, N.M. Lawandy, and J.A. Moon, "Theory of laser action in scattering gain media," Opt. Lett. Vol. 22, pp. 319-321, 1997.

[5] H. Cao, "Lasing in random media," Waves Random Media, Vol. 13, pp. R1-R39, 2003.

[6] M. Leonetti, C. Conti, and C. Lopez, "The mode-locking transition of random lasers," Nature Photon. Vol. 5, pp. 615-617, 2011.

[7] M. Leonetti, C. Conti, and C. Lopez, "Dynamics of phase-locking random lasers," Phys. Rev. A, Vol. 88, pp. 043834 (1-5), 2013.

[8] H. Cao, J.Y. Xu, D.Z. Zhang, S.H. Chang, S.T. Ho, E.W. Seeling, X. Liu, and R.P.H. Chang, "Spatial confinement of laser light in active random media," Phys. Rev. Lett. Vol. 84, pp. 5584-5587, 2000.

[9] H. Cao, J.Y. Xu, S.H. Chang, and S.T. Ho, "Transition from amplified spontaneous emission to laser action in strongly scattering media," Phys. Rev. E, Vol. 61, pp. 19851989, 2000.

[10]H. Cao, Y.G. Zhao, H.C. Ong, S.T. Ho, J.Y. Dai, J.Y. Yu, and R.P.H. Chang, "Ultraviolet lasing in resonators formed by scattering in semiconductor polycrystalline films," Appl. Phys. Lett. Vol. 73, pp. 3656-3658, 1998.

[11] G.D. Dice, S. Mujumdar, and A.Y. Elezzabi, "Plasmonically enhanced diffusive and subdiffusive metal nanoparticle-dye random laser," Appl. Phys. Lett. Vol. 86, pp. 131105 (1-3), 2005.

[12] A.K. Augustine, P. Radhakrishnan, V.P.N. Nampoori, and M. Kailasnath, "Enhanced random lasing from a colloidal CdSe quantum dot-Rh6G system," Laser Phys. Lett. Vol. 12, pp. 025006 (1-4), 2015.

[13]C.J.S. de Matos, L. de S. Menezes, A.M. Brito-Silva, M.A. Martinez-G'amez, A.S.L. Gomes, and C.B. de Ara'ujo, "Random fiber laser," Phys. Rev. Lett. Vol. 99, pp. 153903 (1-4), 2007.

[14]B.H. Hokr, J.N. Bixler, M.T. Cone, J.D. Mason, H.T. Beier, G.D. Noojin, G.I. Petrov, L.A. Golovan, R.J. Thomas, B.A. Rockwell, and V.V. Yakovlev, "Bright emission from a random Raman laser," Nat. Comm. Vol. 5, pp. 4356 (1-5), 2014. 
[15]X. Ma, P. Chen, D. Li, Y. Zhang, and D. Yang, "Electrically pumped $\mathrm{ZnO}$ film ultraviolet random lasers on silicon substrate," Appl. Phys. Lett. Vol. 91, pp. 251109 (1-3), 2007.

[16] B. Redding, M.A. Choma, and H. Cao, "Spatial coherence of random laser emission," Opt. Lett. Vol. 36, pp. 3404-3406, 2011.

[17]F. Luan, B. Gu, A.S.L. Gomes, K. Yong, S. Wen, and P.N. Prasad, "Lasing in nanocomposite random media," Nano Today, Vol. 10, pp. 168-192, 2015.

[18] P.I. Pincheria, A.F. Silva, S.I. Fewo, S.J. Carreño, A.L. Moura, E.P. Raposo, and C.B. de Araújo, "Observation of photonic paramagnetic to spin-glass transition in a specially designed $\mathrm{TiO}_{2}$ particle-based dyecolloidal random laser," Opt. Lett. Vol. 41, pp. 3459-3462, 2016.

[19]D. Zhang, Y. Wang, and D. Ma, "Random lasing emission from a red fluorescent dye doped polystyrene film containing dispersed polystyrene nanoparticles," Appl. Phys. Lett. Vol. 91, pp. 091115 (1-3), 2007.

[20] S. Xiao, T. Li, D. Huang, M. Xu, H. Hu, S. Liu, C. Wang, and T. Yi, "Random laser action from ceramic doped polymer films," J. Modern Opt. Vol. 64, pp. 1289-1297, 2017.

[21]B.R. Anderson, G. Ray, and E. Hergen, "Photodegradation and self-healing in a Rhodamine $6 \mathrm{G}$ dye and $\mathrm{Y}_{2} \mathrm{O}_{3}$ nanoparticledoped polyurethane random laser," Appl. Phys. B, Vol. 120, pp. 1-12, 2015.

[22] B.R. Anderson, G. Ray, and E. Hergen, "Selfhealing organic-dye-based random lasers," Opt. lett. Vol. 40, pp. 577-580, 2015.

[23]B.R. Anderson, G. Ray, and E. Hergen, "Random lasing and reversible photodegradation in disperse orange 11 dyedoped PMMA with dispersed $\mathrm{ZrO}_{2}$ nanoparticles," J. Opt. Vol. 18, pp. 015403 (1-10), 2015.

[24]P.C. de Oliveria, J.A. McGreevy, and N.M. Lawandy, "External-feedback effects in highgain scattering media," Opt. Lett. Vol. 22, pp. 895-897, 1997.

[25] H. Cao, Y.G. Zhao, X. Liu, E.W. Seeling, and R.P.H. Chang, "Effects of external feedback on lasing in random media," Appl. Phys. Lett. Vol. 75, pp. 1213-1215, 1999.

[26]C.T. Dominguez, R.L. Maltez, R.M.S. dos Reis, L.S.A. de Melo, C.B. de Araújo, and A.S.L. Gomes, "Dependence of random laser emission on silver nanoparticle density in PMMA films containing rhodamine 6G," J. Opt. Soc. Am. B, Vol. 28, pp. 1118-1123, 2011.

[27] P. Rafieipour, A.G. Ardakani, and G.M. Parsanasab, "Random lasing emission from FTO and glass substrates coated with dye doped SU-8 epoxy based polymer," Opt. and Laser Tech. Vol. 119, pp. 105602 (1-8), 2019.

[28] P. Rafieipour and A.G. Ardakani, "Fabrication of mechanical controllable, sticky and flexible random lasers based on double-sided tapes," Physica B: Condensed matter, Vol. 574, pp. 411661 (1-9), 2019.

[29]A.S. Wiresma and A. Lagendijk, "Lasing diffusion with gain and random lasers," Phys. Rev. E, Vol. 54, pp. 4256-4265, 1996.

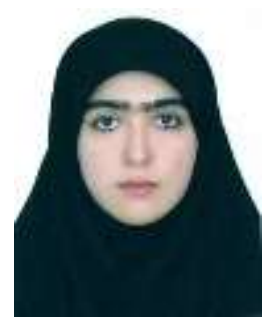

Peymaneh Rafieipour was born in Bushehr, Iran. She received her B.Sc. and M.Sc. in Optics and Laser from Shiraz University, Shiraz, Iran in 2014 and 2016, respectively. Currently, she is a $\mathrm{PhD}$ candidate in Optics and Laser in Shiraz University, Shiraz, Iran. Her current research interest focuses on random lasers.

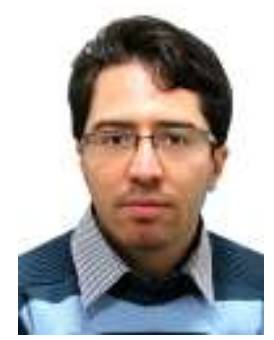

Abbas Ghasempour Ardakani has been an Associate Professor in Physics at Physics 
Department, Shiraz University, Iran since 2012. He has coauthored more than 80 papers in international refereed journals and conferences. His research interests include random lasers, Anderson localization of electromagnetic waves, photonic crystals, Nanophotonics, nanofabrication, Plasmonics, metasurfaces and optical bistability. 
THIS PAGE IS INTENTIONALLY LEFT BLANK. 